\title{
SPIN-WAVE RESONANCE IN MAGNETIC SEMICONDUCTOR THIN FILMS - EXPERIMENT
}

\author{
L.J. Maksymowicz, M. Lubecka and W. Powroźnik \\ Institute of Electronics, Academy of Mining and Metallurgy \\ Al. Mickiewicza 30, Kraków, Poland
}

(Received March 21, 1991)

\begin{abstract}
Magnetic properties of polycrystalline thin films of chromium chalcogenide spinels ( $\mathrm{CdCr}_{2} \mathrm{Se}_{4}$ lightly doped with indium and $\mathrm{CdCr}_{2 x} \operatorname{In}_{2-2 x} \mathrm{Se}_{4}$ ) were studied. The ferromagnetic (FMR) and spin-wave resonance (SWR) techniques were used to investigate the temperature dependences of both the spin-wave stiffness constant $D$ and the saturation magnetization $M_{\mathbf{s}}$. The resonance spectra were recorded in the temperature range extending from $4.2 \mathrm{~K}$ to $300 \mathrm{~K}$. The influence of indium concentration on $M_{\mathrm{s}}(\mathrm{T})$ and $D(T)$ was studied. It was shown that lightly doped samples (In/Cd $<1 \%$ at.) exhibited the ferromagnetic ordering with $M_{\mathrm{s}}(\mathrm{T})$ and $D(T)$ being the linear functions of $T^{3 / 2}$ and $T^{5 / 2}$, respectively. Higher concentration of indium produced the reentrant transition and spin-glass state of magnetic ordering in $\mathrm{CdCr}_{2 x} \mathrm{In}_{2-2 x} \mathrm{Se}_{4}$. The temperature dependence of $M_{s}$ was also found from the FMR data for these two magnetic phases.
\end{abstract}

PACS numbers: $73.60 . \mathrm{Gx}, 75.70 . \mathrm{Ak}$

\section{Introduction}

The magnetic properties of $\mathrm{CdCr}_{2} \mathrm{Se}_{4}$ chalcogenide spinels are very sensitive to In doping $[1,2]$. Our previous results $[3,4]$ show that the magnetic properties of $\mathrm{Cd}-\mathrm{Cr}-\mathrm{In}-\mathrm{Se}$ thin films strongly depend on indium concentration. Chromium spinels of $\mathrm{CdCr}_{2} \mathrm{Se}_{4}: \mathrm{In}$ are classified as magnetic semiconductors. The magnetic properties of these samples indicate the ferromagnetic ordering below the Curie temperature $T_{\mathrm{C}}$. Therefore, the temperature dependences of both the saturation magnetization $M_{\mathrm{s}}$ and the stiffness constant $D$ can be described by the spin-wave theory.

For higher concentration of indium $(0.85 \leq x<1)$ the reentrant transition in $\mathrm{CdCr}_{2 x} \mathrm{In}_{2-2 x} \mathrm{Se}_{4}[5,6]$ is obtained. Further increase in In content $(x<0.85)$ results in the spin-glass behaviour. 
The experimental techniques involved in the measurements of the magnetic properties of thin films require higher sensitivities than standard methods applied in the studies of bulk ferromagnetism. The ferromagnetic (FMR) and spin-wave resonance (SWR) techniques are of particular importance in the studies of the temperature dependences of magnetic parameters.

The SWR spectra with well-resolved volume modes can be obtained by low-energy excitations at the microwave frequency (the microwave spectrometer) for appropriate boundary conditions of surface spins. Then, a dispersion relation for volume modes may be used to calculate the exchange constant $A$ [3]. The saturation magnetization $M_{\mathrm{s}}$ and the stiffness constant $D$ can be derived from the ferromagnetic resonance (FMR) spectra in the perpendicular and parallel geometries.

\section{Experiment}

Thin $\mathrm{CdCr}_{2} \mathrm{Se}_{4}$ films lightly doped with In (In/Cd $<1 \%$ at.) and $\mathrm{CdCr}_{2 x} \operatorname{In}_{2-2 x} \mathrm{Se}_{4}$ with $0.7<x<1$ were deposited in a high vacuum system. Four independent sources of $\mathrm{Cd}, \mathrm{Cr}$, Se and In were used during the evaporation process [7]. Deposition rate was a known function of temperature of the sources. The three-layer structure was prepared as follows. A thin layer of $\mathrm{Cr}(\approx 50 \AA)$ was deposited onto Corning glass substrate preheated to about $520 \mathrm{~K}$ in order to improve the film adhesion. The $\mathrm{Cd}-\mathrm{Cr}-\mathrm{Se}-\mathrm{In}$ film was evaporated at the substrate temperature of about $350 \mathrm{~K}$ onto the $\mathrm{Cr}$ layer. A thin layer of $\mathrm{Cr}$ was then deposited at $350 \mathrm{~K}$ in order to inhibit diffusion of $\mathrm{Cd}$ and Se during the subsequent annealing process. "As-deposited" samples were in the amorphous state. The film thickness was measured after deposition by means of Talysurf 4 profilometer. Thickness of the samples was also controlled during deposition process by the measurement of sample resistivity. The process of crystallization was studied in a Kristalloflex $4 \mathrm{H}$ $\mathrm{X}$-ray diffraction apparatus at different temperatures and for different annealing time intervals. The X-ray diffraction patterns were recorded in situ after each stage of heat treatment had been completed. It was found that the samples reached the polycrystalline state after being annealed at $790 \mathrm{~K}$ for $1 \mathrm{~h}$. The composition of samples was analysed by means of X-ray microprobe (ARL SEMQ microanalyser) and Auger spectroscopy (Riber LAS-620).

The magnetic parameters of the samples, such as the saturation magnetization $M_{\mathrm{s}}, g$-factor, both Curie and Curie-Weiss temperatures, the exchange interaction constant $A$ and the spin-wave stiffness constant $D$, were found from the ferromagnetic resonance (FMR) and spin-wave resonance (SWR). The microwave spectrometer at the X-band was used for FMR and SWR experiments. The spectra were recorded in the temperature range of $4.2-300 \mathrm{~K}$. The external magnetic field was applied in the direction perpendicular to the film plane (perpendicular geometry) and in the direction parallel to the film plane (parallel geometry). 


\section{Results and discussion}

The composition of the samples, i.e. the In doping level was intentionally varied in order to study:

- ferromagnetic state (FM),

- reentrant transition (REE),

- spin-glass state (SG).

\subsection{Ferromagnetic state (FM)}

From the microscopic point of view, FM state is expected at very high spin concentration that yields the long-range spin correlation. The correlated spins form the so-called infinite ferromagnetic network (IFN).

The SWR was detected in thin films of $\mathrm{CdCr}_{2} \mathrm{Se}_{4}$ doped with In $\left(\mathrm{Cd}_{0.99} \mathrm{In}_{0.01} \mathrm{Cr}_{2} \mathrm{Se}_{4}\right)$ in the temperature range from $30 \mathrm{~K}$ to $110 \mathrm{~K}$. It is well-known that the SWR is observed when the surface spins satisfy the appropriate boundary conditions. The semiclassical approach, based on the Rado-Weertman model [8], yields $k=n \pi / L$ for a limiting case of completely pinned surface spins. Here, $k$ is a wave vector of a microwave component of the magnetization, $n$ is an odd integer, and $L$ stands for a film thickness.

The dispersion relation for low-energy excited volume modes in a microwave spectrometry experiment can be expressed as:

$$
\begin{aligned}
& (\omega / \gamma)^{2}=\left(\frac{1}{M_{\mathrm{s}} \sin ^{2} \theta} \frac{\partial^{2} E}{\partial \varphi^{2}}+\frac{2 A}{M_{\mathrm{s}}} k^{2}\right)\left(\frac{1}{M_{\mathrm{s}}} \frac{\partial^{2} E}{\partial \theta^{2}}+\frac{2 A}{M_{\mathrm{s}}} k^{2}\right) \\
& -\left(\frac{1}{M_{\mathrm{s}} \sin \theta} \frac{\partial^{2} E}{\partial \varphi \partial \theta}\right),
\end{aligned}
$$

where $\omega=2 \pi \nu, \nu$ is the microwave frequency and $\gamma$ is the gyromagnetic factor [9]. The density of magnetic anisotropy energy $E(\varphi, \theta)$ depends on the direction of static magnetization $M_{\mathrm{s}}$ (see Fig. 1). The energy is the sum of the following contributions:

$$
E=E_{\mathrm{H}}+E_{\mathrm{d}}+E_{K},
$$

where

$$
E_{\mathrm{H}}=-M_{\mathrm{s}} H\left(\sin \theta \sin \theta_{\mathrm{H}} \sin \varphi+\cos \theta \cos \theta_{\mathrm{H}}\right)
$$

is the energy of magnetic field $H$ applied at $\theta_{\mathrm{H}}, E_{\mathrm{d}}=2 \pi M_{\mathrm{s}}^{2} \cos ^{2} \theta$ is the demagnetization energy, $E_{K}=K \sin ^{2} \theta$ is the energy of uniaxial magnetic anisotropy and $K$ is the magnetic uniaxial anisotropy constant. We set $K=0$ as no magnetic anisotropy showed up in our experiment (polycrystalline state) apart from the 


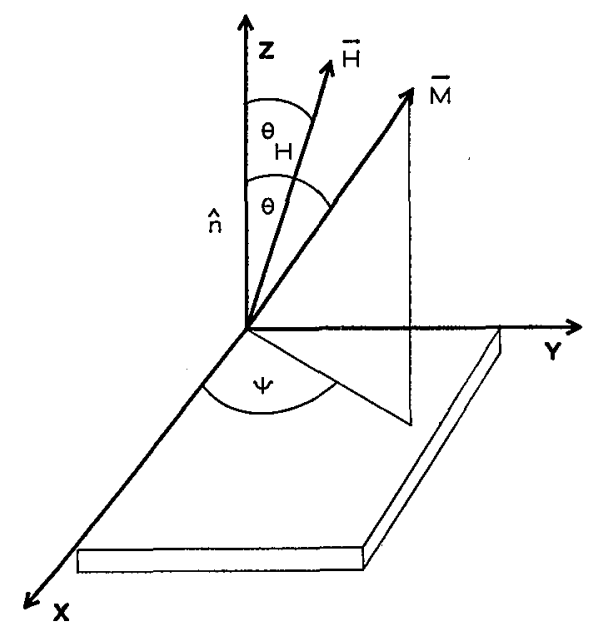

Fig. 1. The coordinate system.

demagnetization contribution. From the equilibrium conditions $\partial E / \partial \varphi=0$ and $\partial E / \partial \theta=0$ one gets $\varphi=\pi / 2$.

For the perpendicular geometry, when $\theta_{\mathrm{H}}=\theta=0$ we have

$$
(\omega / \gamma)=H_{n}-4 \pi M_{\mathbf{s}}+\left(2 A / M_{\mathbf{s}}\right)(n \pi / L)^{2}
$$

and for the uniform mode $(k=0)$

$$
(\omega / \gamma)_{\perp}=H_{\perp}-4 \pi M_{\mathrm{s}} .
$$

In the case of parallel geometry $\theta_{\mathrm{H}}=\theta=90^{\circ}$, hence

$$
(\omega / \gamma)^{2}=\left[H_{n}+\left(2 A / M_{\mathrm{s}}\right)(n \pi / L)^{2}\right]\left[H_{n}+4 \pi M_{\mathrm{s}}+\left(2 A / M_{\mathrm{s}}\right)(n \pi / L)^{2}\right] .
$$

For the uniform mode Eq. (6) reduces to

$$
(\omega / \gamma)_{\|}^{2}=H_{\|}\left(H_{\|}+4 \pi M_{\mathrm{s}}\right)
$$

SWR experiment was performed for the perpendicular geometry, i.e. $\theta_{\mathrm{H}}=\theta=0$. The exchange constant $A$ was found from the best fit of the predicted quadratic dependence of $H_{\mathrm{n}}$ vs. mode number $n$ (see Fig. 2) to the experimental data. In the real case this dependence $\left(H_{n} \propto n^{2}\right)$ cannot be used for lower-order modes because of the microscopic fluctuations of the magnetic parameters.

Figure 2 shows $H_{n}$ as a function of $n^{2}$ for different temperatures. The linear relationship between $H_{n}$ and $n^{2}$ is seen for higher-order modes, thus allowing to establish the value of $\left(2 A / M_{\mathrm{s}}\right)(\pi / L)^{2}$ from the slope of the line. The temperature dependences of the resonance field $H_{\mathrm{r}}$ of the uniform mode in the perpendicular and parallel geometries are presented in Fig. 3. Ferromagnetic-paramagnetic transition temperature, i.e. the temperature at which $H_{\|}=H_{\perp}$, can be determined 


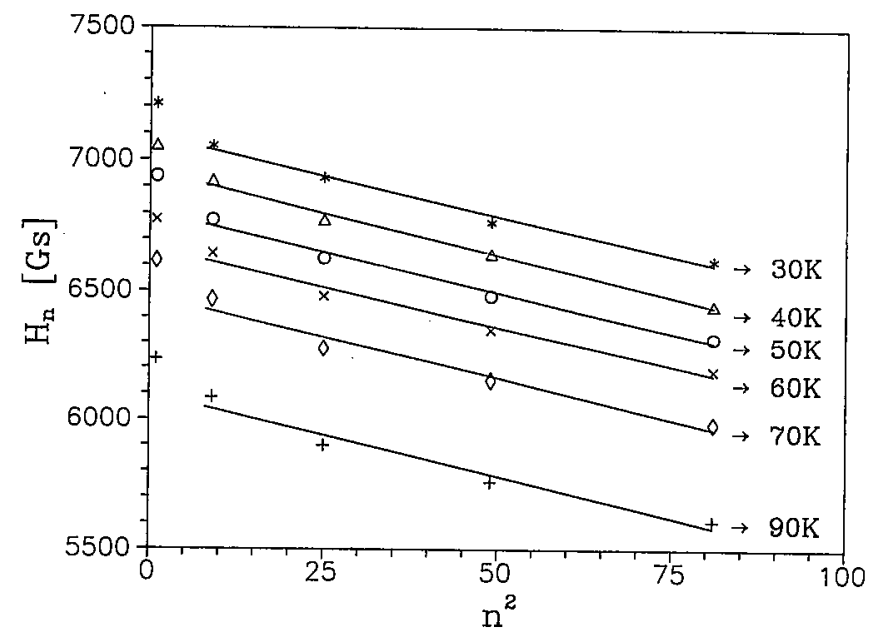

Fig. 2. The dependence of the resonance-field position $H_{n}$ on the square of the mode number $n^{2}$ for $5000 \AA$-thick $\mathrm{CdCr}_{2} \mathrm{Se}_{4}$ :In sample. Solid lines represent the best linear fit to the experimental data ( $n=1$ was excluded from the fitting procedure). $A=$ $(2.3 \mp 0.2) \times 10^{-12} \mathrm{~J} / \mathrm{m}$ for $T=40 \mathrm{~K}, A=(2.4 \mp 0.2) \times 10^{-12} \mathrm{~J} / \mathrm{m}$ for $T=50 \mathrm{~K}$.

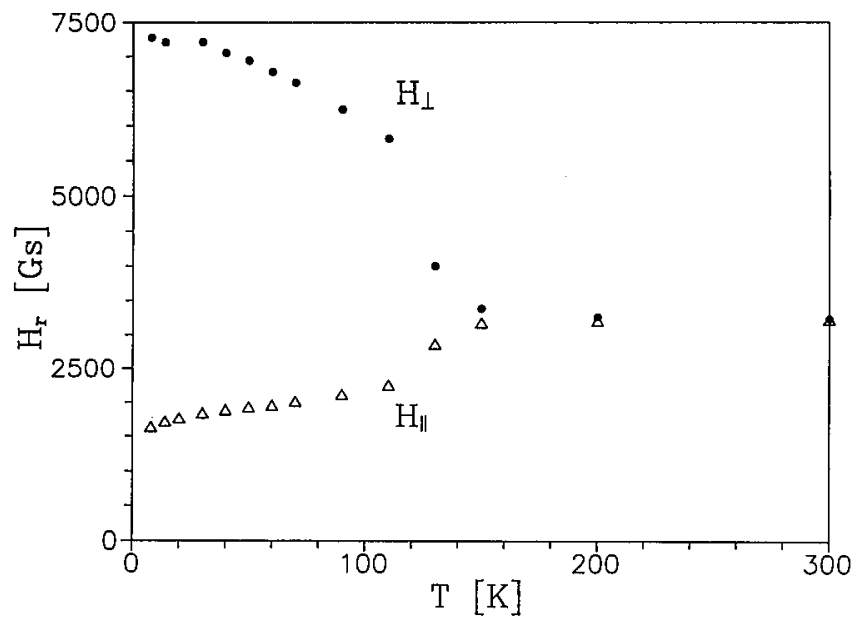

Fig. 3. The temperature dependence of the resonance field $H_{\mathrm{r}}$ for $\mathrm{CdCr}_{2} \mathrm{Se}_{4}$ :In (perpendicular geometry $H_{\perp}$, parallel geometry $\left.H_{\|}\right)$.

easily from these data. From Eqs. (5) and (7), assuming that $(\omega / \gamma)_{\perp}=(\omega / \gamma)_{\|}$, one gets

$$
4 \pi M_{\mathrm{s}}=H_{\perp}+0.5 H_{\|}-\left[H_{\|}\left(H_{\perp}+1.25 H_{\|}\right)\right]^{1 / 2} .
$$


The temperature dependence of $M_{\mathrm{s}}$ is obtained from the experimental data (Fig. 3 and Eq. 8) presented above. Figure 4 shows $M_{\mathrm{s}}$ vs. $T^{3 / 2}$ for $\mathrm{CdCr}_{2} \mathrm{Se}_{4}$ thin film lightly doped with In. It is seen that the Bloch's law holds. From the spin-wave theory [10] one expects

$$
M_{\mathrm{s}}(T)=M(0)\left(1-B T^{3 / 2}-\ldots\right) .
$$

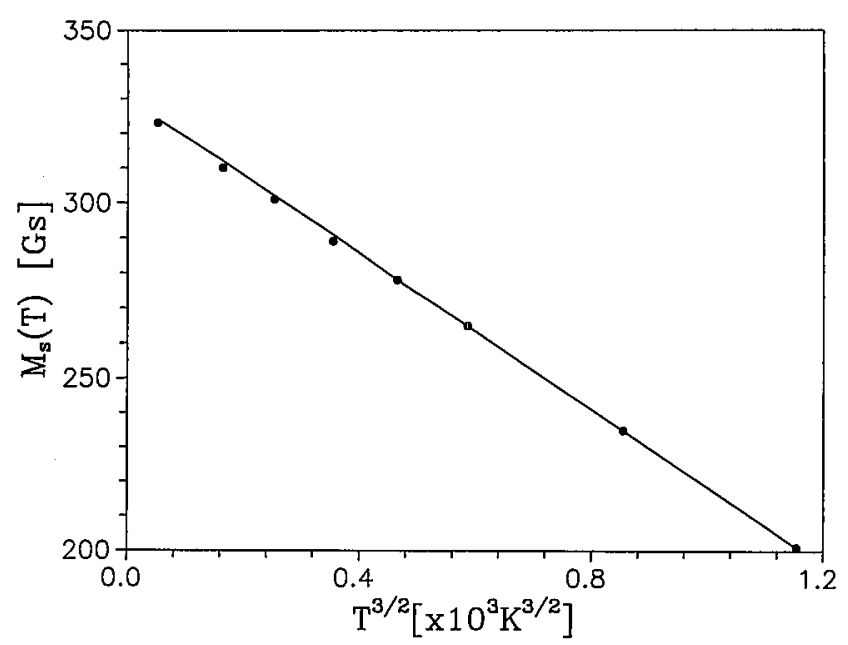

Fig. 4. The temperature dependence of the saturation magnetization $M_{\mathrm{s}}(T)$ for $\mathrm{CdCr}_{2} \mathrm{Se}_{4}$ :In. Solid line represents the best fit of Eq. (9).

The spin-wave stiffness constant $D$ is related to $B$ :

$$
B=\xi(3 / 2)\left(g \mu_{\mathrm{B}} / M(0)\right)\left(k_{\mathrm{B}} / 4 \pi D\right)^{3 / 2},
$$

where $\xi$ stands for Riemann $\xi$-function and $k_{\mathrm{B}}$ is the Boltzmann constant.

The value of $B$ determined from the experimental data on $M$ vs. $T^{3 / 2}$ was used to calculate $D$ from Eq. (10). We obtained $D(0)=31.29 \mathrm{meV} \AA^{2}$. Figure 5 presents $\Delta D(T) / D(0)$ vs. $T^{5 / 2}$ calculated from the SWR data for the same sample. The stiffness constant $D$ scales as $T^{5 / 2}$ according to the spin-wave theory [11].

\subsection{Reentrant transition (REE)}

The reentrant transition is expected if a spin concentration is as high as required for the onset of the ferromagnetic spin ordering. The medium could be treated as consisting of an infinite ferromagnetic network (IFN) and finite spin clusters (FC). The intracluster interactions are stronger than intercluster coupling. Below the freezing temperature $T_{\mathbf{f}}$ the ferromagnetic state (FM) becomes unstable and evolves into a spin-glass state (SG). 


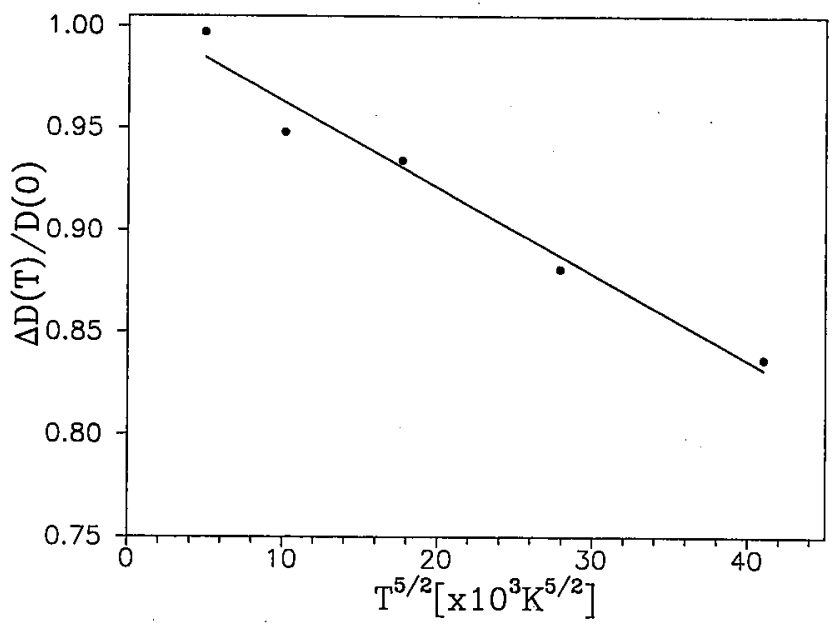

Fig. 5. The temperature dependence of the spin-wave stiffness constant $\Delta D(T) / D(0)$ for $\mathrm{CdCr}_{2} \mathrm{Se}_{4}: \mathrm{In} ; \Delta D(T)=D(0)-D(T)$. Solid line represents the linear fit to the experimental data.

The microscopic picture of this SG gives FC embedded in the sea of weakly coupled spins. In this state the effect of disorder should be the most significant. The phenomenological explanation of the double transition (REE) is still far from being clear and settled.

The REE transition alters the temperature dependences of the basic magnetic parameters, hence it affects the FMR and SWR resonance data. In the films with the REE one expects to find the SWR only above the freezing temperature $T_{\mathrm{f}}$, while at $T<T_{\mathrm{f}}$ the uniform mode is detected. It is the case for thin films $\mathrm{CdCr}_{1.9} \mathrm{In}_{0.1} \mathrm{Se}_{4}$. Figures $6 \mathrm{a}$ and $6 \mathrm{~b}$ present the uniform mode below $T_{\mathrm{f}}$ and $\mathrm{SWR}$ modes above $T_{\mathrm{f}}$, respectively. The exchange interaction constant $A$ was calculated from the positions of volume modes (see Eq. (4)) above $T_{\mathrm{f}}$. The value of $A=(1.6 \mp 0.2) \times 10^{-12} \mathrm{~J} / \mathrm{m}$ at $T=100 \mathrm{~K}$ is close to that determined in the $\mathrm{FM}$ state (see Fig. 6). It should be emphasized that the results of calculations are more reliable at higher temperatures because the modes become well-resolved.

The temperature dependence of the saturation magnetization $M_{\mathbf{s}}(\mathrm{T})$ was obtained from the FMR data using Eq. (8). As it is seen in Fig. 7, the experimental $M_{\mathbf{s}}(\mathrm{T})$ deviates from $T^{3 / 2}$ for $T<T_{\mathfrak{f}}\left(T_{\mathfrak{f}} \approx 50 \mathrm{~K}\right)$.

The experimental results presented above can be interpreted on the basis of the model proposed in $[12,13]$. The model invokes an energy gap $\Delta_{\mathrm{r}}$ in the dispersion relation for magnons $E=\Delta_{\mathrm{r}}+D k^{2}$. It modifies the density of states and yields a non-zero density of states at the energy gap which is the main feature of REE at low temperatures. The standard statistical mechanics for bosons predicts 

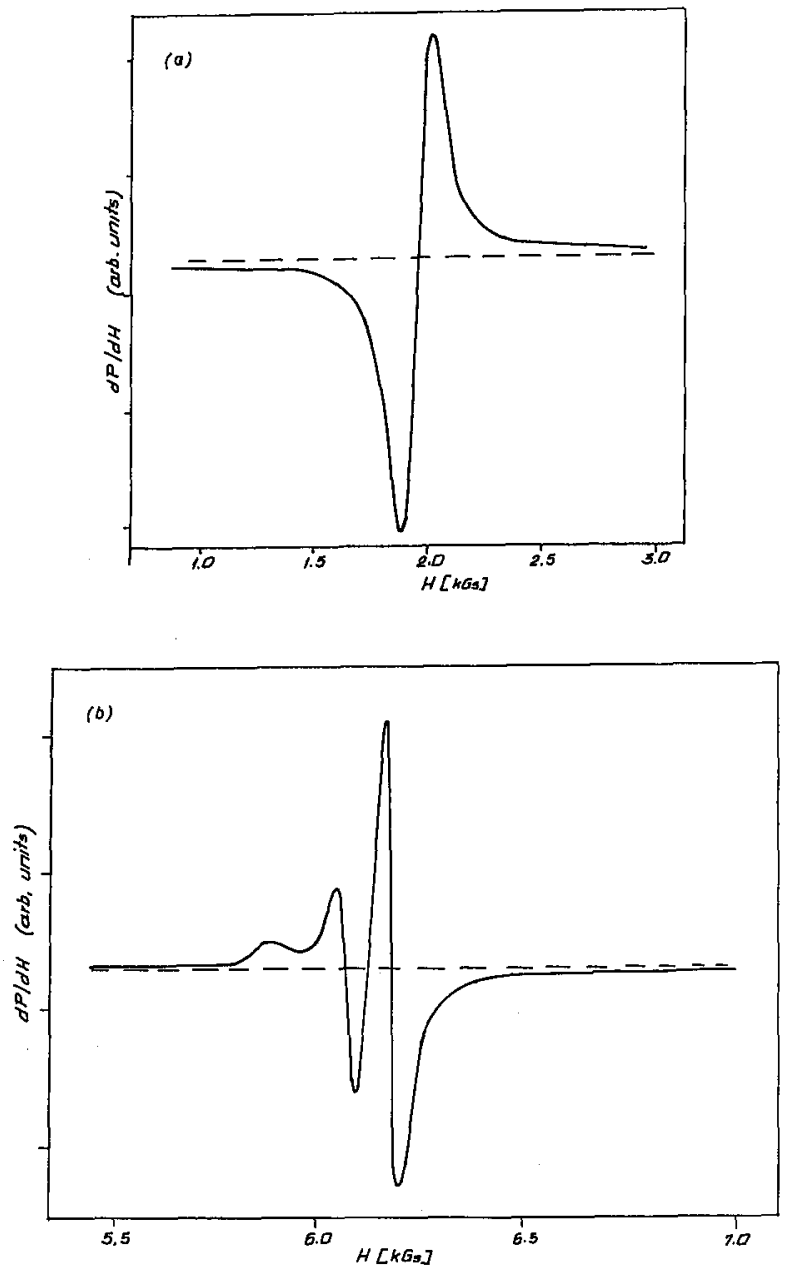

Fig. 6. Spin-wave resonance spectra for $\mathrm{CdCr}_{1.9} \operatorname{In}_{0.1} \mathrm{Se}_{4}$ thin film at (a) $T \leq T_{\mathrm{f}}$ and (b) $T>T_{\mathrm{f}}$.

in this case [12]:

$$
\left[M(0)-M_{\mathbf{s}}(T)\right] / M(0)=B T^{3 / 2} / \xi(3 / 2) \sum_{n=1}^{\infty}\left[\exp \left(-n \Delta_{\mathrm{r}} / k_{\mathrm{B}} T\right) / n^{3 / 2}\right]
$$

According to Eq. (11), the deviation from the classical $T^{3 / 2}$ law becomes more pronounced as the energy gap increases. The two-parameter fitting procedure based on Eq. (11) was employed and its results are demonstrated in Fig. 7. $M(0)$ was taken from the extrapolation of the FMR data; $\Delta_{\mathrm{r}}$ and $B$ were treated as fitting parameters. Figure 8 shows that Eq. (11) works quite well above the character- 


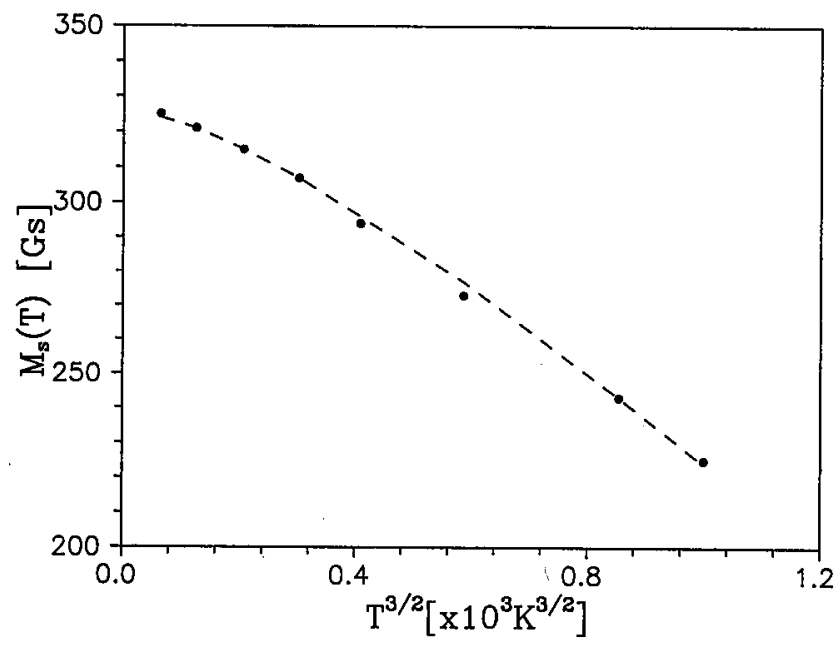

Fig. 7. $M_{\mathrm{s}}(T)$ vs. $T^{3 / 2}$ for $\mathrm{CdCr}_{1.9} \operatorname{In}_{0.1} \mathrm{Se}_{4}$ thin film. Solid line represents the best fit to Eq. (11). $\Delta_{\mathrm{r}}=5 \mathrm{~K}, T_{\mathrm{f}}=50 \mathrm{~K}$.

istic temperature $T_{\mathrm{f}}$ as predicted in [12]. At low temperatures $\left(T<T_{\mathrm{f}}\right)$, where one expects the breakdown of IFN, the model assumes $\delta$-like density of states $\rho(E)=\delta\left(E-\Delta_{\mathrm{r}}\right)$ that results in a completely different behaviour of $M_{\mathbf{s}}(\mathrm{T})$ [13].

\subsection{Spin-glass state (SG)}

The concentrated spin-glass state (SG) could be microscopically illustrated as a state in which there is no IFN at all. Therefore, in the whole temperature range extending from the Weiss temperature $\Theta_{\mathrm{p}}$ down to $T_{\mathrm{SG}}$, which represents the characteristic temperature of spin freezing in a random direction, the dispersion relation for magnons can be expressed as $E=\Delta_{\mathrm{s}}$ with $\Delta_{\mathrm{s}}$ being the energy gap. Low-spin concentration produces a strong interaction fluctuation which becomes a source of the SG phase. Theoretical understanding of such behaviour and its consequences is far from being complete.

In the SG state the temperature dependence of saturation magnetization differs distinctly from that observed in the ferromagnetic state and reentrant systems. At $T>T_{\mathrm{SG}}$ the linear relationship between $M$ and $T$ is obtained while below $T_{\mathrm{SG}}$ $M_{\mathrm{s}}$ is $T$-independent. Following the arguments presented in $[12,13]$, the non-zero density of states is expected at $\Delta_{\mathrm{s}}$ only. In the phenomenological picture of concentrated SG it corresponds to the breakdown of the IFN which provides for the development of separate, non-interacting spin clusters.

Thus, the temperature dependence of saturation magnetization takes the form:

$$
\left[M(0)-M_{\mathrm{s}}(T)\right] / M(0)=C_{\mathrm{s}} /\left[\exp \left(\Delta_{\mathrm{s}} / k_{\mathrm{B}} T\right)-1\right],
$$




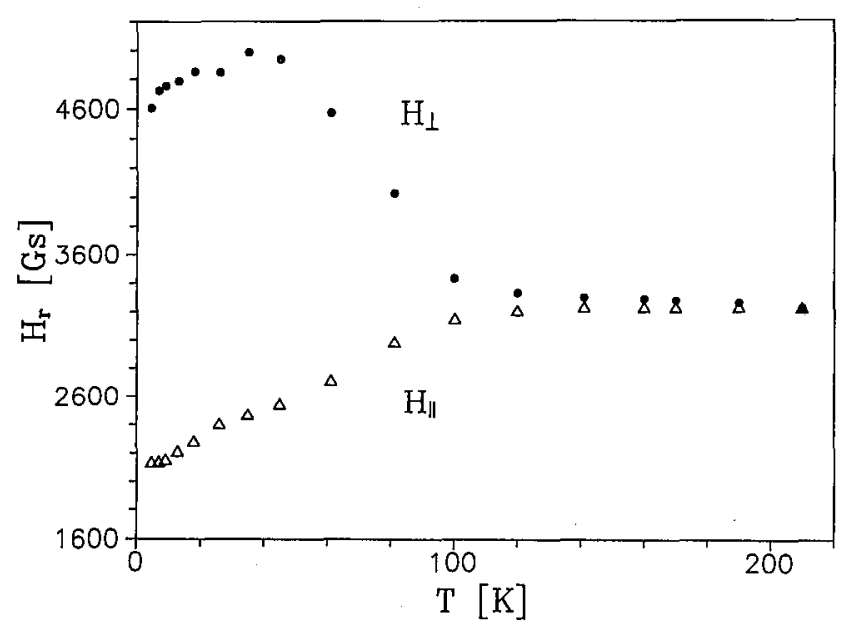

Fig. 8. The temperature dependence of resonance field $H_{\mathrm{r}}$ for $\mathrm{CdCr}_{1.6} \operatorname{In}_{0.4} \mathrm{Se}_{4}$ thin film.

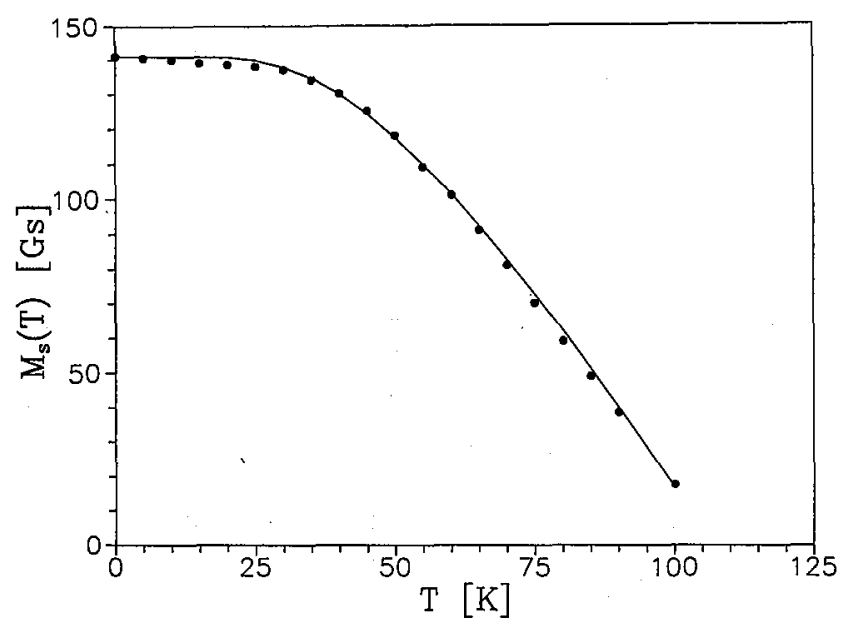

Fig. 9. The temperature dependence of $M_{s}(T)$ for $\mathrm{CdCr}_{1.6} \operatorname{In}_{0.4} \mathrm{Se}_{4}$ thin film. Solid line represents the best fit to Eq. (12). $\Delta_{s}=145 \mathrm{~K}, C_{\mathrm{s}}=2.9$.

where $C_{\mathrm{s}}$ is responsible for the density of states at $\Delta_{\mathrm{s}}$ and $\Delta_{\mathrm{s}}$ is a measure of the intracluster interaction. The same analytical formula as presented in Eq. (12) is valid for $\mathrm{REE}$ at $T<T_{\mathrm{f}}$, but the energy gap is addressed as $\Delta_{\mathrm{s}}$. It seems important to point out that, in general, $\Delta_{\mathrm{s}}$ is larger than $\Delta_{\mathrm{r}}$ and closer to the Weiss temperature $\Theta_{\mathrm{p}}$. 
The temperature dependences of the resonance field $H_{\mathrm{r}}$ of the uniform mode for thin $\mathrm{CdCr}_{1.6} \mathrm{In}_{0.4} \mathrm{Se}_{4}$ film are presented in Fig. 8.

Figure 9 shows the experimental data on $M_{\mathrm{s}}$ vs. $T$ for this film along with the numerical results given by Eq. (12). From the best fit to the experimental data two fitting parameters $\Delta_{\mathrm{s}}$ and $C_{\mathrm{s}}$ were obtained.

\section{Conclusions}

In this paper the magnetic properties of thin polycrystalline films of $\mathrm{CdCr}_{2 x} \operatorname{In}_{2-2 x} \mathrm{Se}_{4}$ and $\mathrm{CdCr}_{2} \mathrm{Se}_{4}$ lightly doped with In were discussed. The magnetic properties of these films were found to be very sensitive to any deviation from stoichiometry and in particular to In concentration. Three different magnetic types of spin ordering, i.e. the ferromagnetic state $\mathrm{FM}$, the reentrant transition (REE) and the spin-glass state (SG) were obtained in a series of samples with the intentionally varied In content (for $0.80 \leq x \leq 1.0$ ).

We have identified the ferromagnetic state in thin films of $\mathrm{CdCr}_{2} \mathrm{Se}_{4}$ lightly doped with In. The FMR and SWR experiments carried out at X-band in the temperature range of $4.2 \mathrm{~K} \leq T \leq 150 \mathrm{~K}$ allowed to determine the temperature dependences of the saturation magnetization and spin-wave stiffness constant using the boundary conditions for completely pinned surface spins.

The analytical description of the SWR and FMR was based on the semiclassical approach. We have found that the temperature dependence of the saturation magnetization $M_{\mathrm{s}}(\mathrm{T})$ obeys the Bloch's law. Thin $\mathrm{CdCr}_{2 x} \operatorname{In}_{2-2 x} \mathrm{Se}_{4}$ films with $x=0.95$ exhibit reentrant transition. The temperature dependence of saturation magnetization in that case distinctly deviates from the behaviour given by the spin-wave model. The model proposed in $[12,13]$ was used to account for the observed behaviour of $M_{\mathrm{s}}$. The dispersion relation of magnons was modified by including the energy gap $\Delta_{\mathrm{r}}$ with non-zero density of state. Therefore, the analytical expression for the deviation from $T^{3 / 2}$ law was found (see Eq. (11)). It was demonstrated that Eq. (11) satisfactorily fitted our experimental data on $M_{\mathrm{s}}(\mathrm{T})$ (see Fig. 7). We also observed that for $T>T_{\mathrm{f}}$ the volume modes were excited and their separation increased with temperature. The exchange interaction constant $A$ was then derived.

The increase in In concentration resulted in the enhanced degree of spin disorder. The spin-glass state was achieved in thin $\mathrm{CdCr}_{2 x} \mathrm{In}_{2-2 x} \mathrm{Se}_{4}$ films with $x=0.8$. The temperature dependence of saturation magnetization for samples in SG state significantly differs from that characteristic for FM and REE. The saturation magnetization $M_{\mathrm{s}}$ is constant at low temperatures and reduces as $T$ for $T>T_{\mathrm{SG}}$, where $T_{\mathrm{SG}}$ is a measure of intercluster interaction. The analytical formula for $M_{\mathrm{s}}(\mathrm{T})$ predicted in this case exploits only localized states at the energy gap $\Delta_{\mathrm{s}}$ (Eq. (12)). The energy gap $\Delta_{\mathrm{s}}$ is responsible for the intracluster interaction. The satisfactory agreement between the experimental and numerical data (see Fig. 9) was obtained. The value of $T_{\mathrm{SG}}$ was found from the temperature dependence of the resonance field while $\Delta_{\mathrm{s}}$ was used as a fitting parameter (see Fig. 9).

Finally, we can conclude that in the case of the magnetic semiconductor thin $\mathrm{Cd}-\mathrm{Cr}-\mathrm{Se}-\mathrm{In}$ films the amount of In, very carefully controlled during deposition 
process, significantly modifies the magnetic properties of the material. The FMR and SWR techniques are suitable tools in the studies of the magnetic properties of such systems including coupling between spin clusters as well as the intracluster interactions.

\section{Acknowledgments}

The authors are grateful to Dr. R. Żuberek from the Institute of Physics, Polish Academy of Sciences in Warsaw for the FMR measurements and also to Dr. K. Zakrzewska from the Institute of Electronics, AMM and Dr. A. Maksymowicz from the Institute of Computer Science, AMM for their contribution to the numerical analysis.

This work was partly supported by the Project CPBP 01.08.

\section{References}

[1] H.L. Pinch, S.B. Berger, J. Phys. Chem. Solids 29, 2091 (1968).

[2] M. Alba, J. Hammann, M. Nogues, J. Phys. C, Solid State Phys. 15, 5441 (1982).

[3] M. Lubecka, L.J. Maksymowicz, R. Żuberek, W. Powroźnik, Phys. Rev. B 40, 2097 (1989).

[4] M. Lubecka, L.J. Maksymowicz, R. Żuberek, Phys. Rev. B 42, 3926 (1990).

[5] S. Viticoli, D. Fiorani, M. Nogues, J.L. Dormann, Phys. Rev. B 26, 6085 (1982).

[6] D. Fiorani, M. Nogues, S. Viticoli, Solid State Commun. 41, 537 (1982).

[7] M. Lubecka, W. Powroźnik, Thin Solid Films 174, 71 (1989).

[8] C. Kittel, Phys. Rev. 110, 1295 (1958).

[9] A. Maksymowicz, Phys. Rev. B 33, 6045 (1986).

[10] F. Keffer, Handbuch der Physik, Ed. S. Fluege, Springer-Verlag, Berlin 1966, Vol. 18, Part 2, p. 1.

[11] J.A. Tarvin, G. Shirane, R.J. Birgeneau, H.S. Chen, Phys. Rev. B 17, 241 (1978).

[12] E.M. Jackson, S.B. Liao, S.M. Bhagat, M.A. Manheimer, J. Magn. Magn. Mater. 80, 229 (1989).

[13] S.B. Liao, S.M. Bhagat, M.A. Manheimer, K. Moorjani, J. Appl. Phys. 63, 4354 (1988). 